\title{
Efeitos de Expectativas de Futuro no Comprometimento Organizacional e Intenção de Desligamento
}

\author{
Vinicius Vasconcellos* \& Elaine Rabelo Neiva \\ Universidade de Brasília, Brasília, DF, Brasil
}

\begin{abstract}
RESUMO - Expectativas constituem parte relevante na vida laboral, mas seus efeitos em outras variáveis de comportamento organizacional continuam pouco explorados. Assim, esta pesquisa testou modelo no qual expectativas de futuro organizacional e de carreira na organização atuavam como antecedentes de comprometimento organizacional afetivo e intenção de desligamento. Profissionais de diversas organizações $(n=370)$ responderam a escalas validadas no Brasil. Expectativas de futuro organizacional relacionaram-se com comprometimento organizacional afetivo e expectativas de carreira na organização. Essa última variável atuou como antecedente de comprometimento organizacional afetivo e intenção de desligamento. $\mathrm{O}$ tipo de organização e a orientação para o futuro dos respondentes atuaram como moderadores no modelo. Discute-se, então, a dimensão temporal da dinâmica indivíduo-organização e sua importância para a gestão de pessoas.
\end{abstract}

PALAVRAS-CHAVE: desenvolvimento profissional, expectativas, comprometimento organizacional, rotatividade de pessoal

\section{Effects of Future Expectations on Organizational Commitment and Intention to Leave}

\begin{abstract}
Expectations are a relevant part of working life, but their effect on other variables in the field of organizational behavior remain poorly explored. Thus, this research tested a model in which organizational future expectations and career expectations in the organization acted as antecedents of affective organizational commitment and intention to quit. Professionals from several organizations $(n=370)$ answered scales validated in Brazil. Organizational future expectations were related to affective organizational commitment and career expectations in the organization. The latter variable acted as an antecedent of affective organizational commitment and intention to quit. The organization's sector and the future expectations of the participants act as moderates in the model. The discussion analyzes the temporal dimension of the individual-organization relationship and its importance to human resource management.
\end{abstract}

KEYWORDS: professional development, expectations, organizational commitment, employee turnover

A capacidade de se relacionar com o futuro figura-se como aspecto relevante da subjetividade humana. Não é por acaso que modelos e teorias de grande vulto na psicologia concederam às expectativas e percepções de futuro papel notável na motivação e na produção/mudança de comportamento (Bandura, 1977; Vroom, 1964). Mais recentemente, estudos sublinham como a prospecção de futuros possíveis é peça central na cognição, afeto, motivação e ação dos indivíduos (Seligman, Railton, Baumeister, \& Sripada, 2013) e como o otimismo influencia diversos aspectos da experiência humana (Carver \& Scheier, 2017). Partindo dessas contribuições seminais, apreende-se que o presente é condicionado pelas projeções de futuro formuladas pelos os indivíduos.

Esta pesquisa aborda, especificamente, o modo como o presente e o futuro se entrelaçam nas organizações contemporâneas. Com efeito, a aceleração dos avanços tecnológicos e a volatilidade nos mercados nas últimas décadas ensejaram rápidos fluxos de mudança no contexto organizacional, de modo que o futuro assumiu alto grau de incerteza. Tal conjuntura de incerteza repercute não apenas nas projeções de futuro das organizações, mas também na forma como os indivíduos lidam com a carreira, entendida aqui como a sequência de experiências relacionadas ao

*E-mail: viniciuscarvalhodevasconcellos@gmail.com

- Submetido: 03/11/2015; Revisado: 16/02/2018; Aceito: 01/08/2018. 
trabalho no curso da vida do profissional (Greenhaus \& Kossek, 2014). Nesse contexto, a carreira passa a ser cada vez mais concebida como projeto aberto e imprevisível. Atualmente, as decisões sobre o futuro na carreira estão entre as principais fontes de preocupação e interesse dos profissionais (Magalhães \& Bendassolli, 2013).

Para abordar o enlace presente-futuro na cena organizacional, este artigo centra-se em expectativas, isto é, crenças sobre o futuro provável (Oettingen \& Mayer, 2002), mais especificamente, nas expectativas dos indivíduos sobre sua carreira e sobre o futuro de sua organização. O objetivo da pesquisa é testar um modelo que propõe esses dois tipos de expectativas (variáveis orientadas para o futuro) como antecedentes de comprometimento organizacional afetivo e intenção de desligamento (variáveis orientadas para o presente), avaliando também possíveis moderadores.
Investigações anteriores, malgrado a variabilidade de medidas e construtos adotados, relataram que projeções de futuro organizacional e de carreira correlacionam-se a diferentes atitudes e comportamentos no ambiente organizacional (Chay \& Aryee, 1999; Chiu, 2002; Prince, 2003). Assim, variáveis orientadas para o futuro parecem conformar área promissora de estudo, embora essa literatura ainda seja incipiente e fragmentada. No fundo, a dimensão temporal (não apenas o futuro) tende a ser ignorada ou posta em segundo plano na literatura de organizações e trabalho (Lee \& Liebenau, 1999; Sonnentag, 2012).

Ciente dessa lacuna, esta pesquisa aprecia o papel da temporalidade (em especial das expectativas de futuro), no seio da relação indivíduo-organização. A próxima seção detalha o modelo proposto e suas hipóteses.

\section{MODELO DE INVESTIGAÇÃO PROPOSTO E DESENVOLVIMENTO DE HIPÓTESES}

A Figura 1 expõe o modelo de investigação proposto. Esse modelo testa o impacto das expectativas de futuro organizacional e de carreira no comprometimento organizacional afetivo e na intenção de desligamento.

Com exceção da associação entre comprometimento organizacional e intenção de desligamento, poucos estudos exploraram empiricamente as relações propostas no modelo e, quando o fizeram, relataram efeitos isolados para cada par de variáveis. Em seu desenho, o modelo permite analisar, de forma integrada, as diversas relações entre variáveis. Ademais, tipo de organização do profissional e orientação para o futuro são examinados como potenciais moderadores da relação dos dois tipos de expectativas com as duas principais variáveis dependentes do estudo (comprometimento organizacional afetivo e intenção de desligamento), iniciativa que não foi localizada na literatura.

A rigor, a proposição/teste do modelo atende aos apelos de artigos teóricos sobre carreira e desligamento, que convocam mais pesquisas com variáveis orientadas para o futuro (Lawrence \& Tolbert, 2007; Mitchell, Burch, \& Lee, 2014). As hipóteses, forjadas com base em estudos pregressos de comportamento organizacional, psicologia social e psicologia do tempo, são caracterizadas nas próximas subseções.

\section{Expectativas de Futuro Organizacional e de Carreira na Organização}

No modelo, a relação dos indivíduos com o futuro traduz-se em duas variáveis que aferem expectativas. A primeira, expectativas de futuro organizacional, trata das crenças dos profissionais sobre a gestão e a efetividade de sua organização no futuro (Vasconcellos \& Neiva, 2017). Como está no nível individual, tal construto distingue-se de construtos vinculados ao planejamento, como visão da

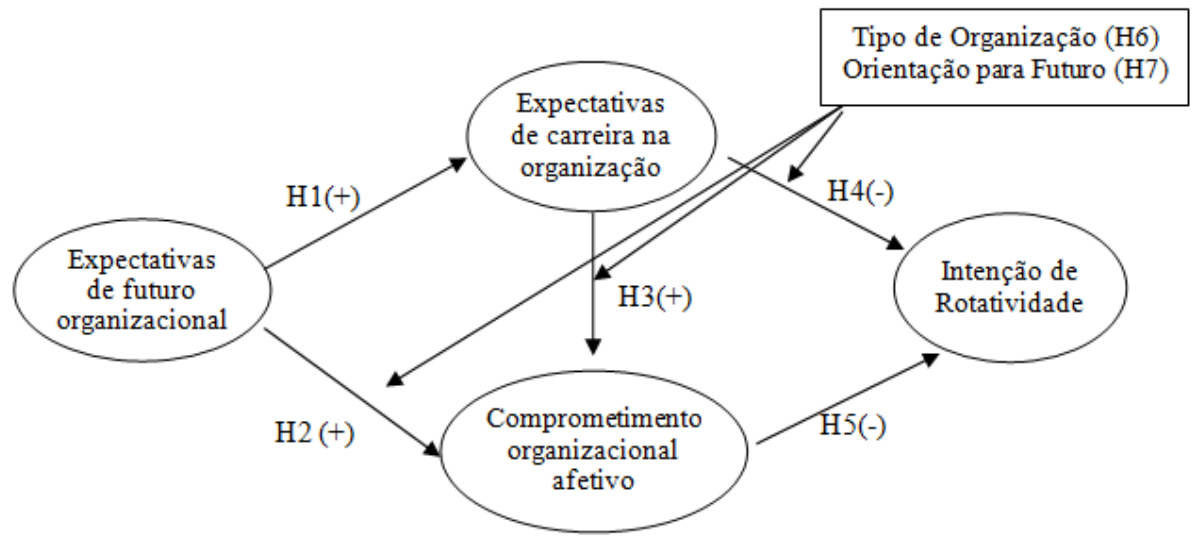

Figura 1. Modelo de investigação proposto. 
organização e cenários prospectivos. A segunda variável, expectativas de carreira na organização, expressa as crenças dos indivíduos sobre sua futura trajetória profissional na atual organização (Vasconcellos \& Neiva, 2016). Ao abordar expectativas, essa variável diferencia-se das variáveis de carreira orientadas para o presente (satisfação com a carreira, por exemplo) ou para o futuro desejado, preferido ou ideal (aspirações/objetivos de carreira).

Ao pensar sobre sua futura carreira na organização, parece plausível supor que os indivíduos considerem o futuro dessa última. Profissionais que vislumbram perspectivas favoráveis para a organização - como expansão nas operações e aumento no lucro - podem presumir que tais perspectivas se desdobrarão em oportunidades ou benesses para eles mesmos. Pesquisas qualitativas durante mudanças organizacionais já identificaram a vinculação entre expectativas de carreira e de futuro organizacional (Duarte \& Silva, 2013; Margolis \& Hansen, 2003; Ullrich, Wieseke, \& van Dick, 2005).

Nessas investigações, expectativas sobre crescimento dos negócios e melhorias na imagem da organização vincularam-se a expectativas de avanços na carreira e de aumento nos salários/benefícios. Por outro lado, incertezas sobre a estrutura organizacional e o ambiente de trabalho no futuro atrelaram-se à insegurança sobre o próprio futuro profissional. Esses resultados sugerem relação positiva entre os dois conjuntos de expectativas. Neste estudo, a relação entre essas variáveis será testada por meio de medidas quantitativas, proporcionando um olhar distinto, conquanto complementar às pesquisas citadas. Assim, a primeira hipótese determina que:

Hipótese 1 - Expectativas de futuro organizacional relacionam-se positivamente às expectativas de carreira na organização.

\section{Expectativas de Futuro Organizacional e Comprometimento Organizacional Afetivo}

O modelo também propõe que expectativas de futuro organizacional se associam ao comprometimento organizacional afetivo. Esse comprometimento se refere a um vínculo entre indivíduo e organização, que expressa o sentimento de gostar e de identificação (Menezes \& Bastos, 2011).

Sobre o questionamento "De que forma estas variáveis podem se relacionar?", Seligman et al. (2013), em artigo teórico, frisam como prospecções e expectativas ligamse a valências afetivas. Assim, expectativas de futuro organizacional favoráveis podem favorecer a construção de vínculos afetivamente positivos. Por outro lado, no cenário de um futuro organizacional pouco alvissareiro, a organização poderia se tornar um lugar arriscado para se trabalhar a médio e longo prazo, minando o comprometimento. Estudos anteriores apontam nessa mesma direção. Mossholder, Settoon, Harris e Armenakis (1995) detectaram correlação positiva entre comprometimento organizacional e otimismo quanto ao futuro da organização, variável aferida a partir de uma escala que continha apenas dois itens. Por sua vez, Chiu (2002) relata que percepções sobre o desenvolvimento futuro da organização operam como antecedentes de comprometimento afetivo (relação positiva).

De acordo com Chiu (2002), esta pesquisa enfatiza o componente afetivo do comprometimento. Todavia, adota-se, aqui, forma distinta de conceituar/aferir o futuro organizacional na perspectiva dos indivíduos, oferecendo à literatura teste alternativo da relação entre as variáveis. De todo modo, há na literatura base suficiente para a segunda hipótese:

Hipótese 2 - Expectativas de futuro organizacional relacionam-se positivamente ao comprometimento organizacional afetivo.

\section{Expectativas de Carreira na Organização, Comprometimento Organizacional Afetivo e Intenção de Desligamento}

O modelo prevê, ainda, expectativas de carreira na organização como preditor de comprometimento organizacional afetivo (relação positiva) e de intenção de desligamento (relação negativa). Essa última reflete a intenção individual (consciente e deliberada) do trabalhador em sair (se desligar) de sua atual organização, sendo também chamada, na literatura, de forma intercambiável, de intenção de rotatividade (Cho, Johanson, \& Guchait, 2009). Como o termo rotatividade também é rotineiramente utilizado para designar variável de nível organizacional, que retrata o balanço entre admissões e demissões, optou-se, aqui, por preservar o termo intenção de desligamento.

As hipóteses citadas no parágrafo anterior apoiam-se em dois alicerces: a teoria da troca social e a norma da reciprocidade, ambas com raízes nas ciências sociais e na psicologia social. A primeira pontua que trocas sociais representam um processo de interação fundado na confiança, gratidão e interdependência dos envolvidos, no qual favores, contribuições e benefícios ofertados por uma das partes (por exemplo, a organização) desperta na outra parte (por exemplo, o profissional) a obrigação de retribuir (Blau, 1964). No mesmo campo semântico, a norma da reciprocidade é concebida como componente moral que define, como obrigação, o ato de retribuir um benefício recebido (Gouldner, 1960). O ato de reciprocidade não emerge de exigência legal, mas do anseio de reequilibrar a relação social.

Nessa linha, expectativas favoráveis de carreira podem ser assumidas pelos indivíduos como um benefício ofertado pela organização, o que ensejaria a necessidade de retribuição por meio de alto comprometimento e baixa intenção de desligamento. Constitui-se, assim, uma relação interdependente e selada na confiança recíproca. Em contraste, se há expectativas de carreira negativas, a troca 
social e a reciprocidade cessam, levando à diminuição no comprometimento e ao crescimento na intenção de desligamento.

No caso da relação entre expectativas de carreira e comprometimento organizacional, outras fontes podem ser evocadas para embasar a hipótese. Por exemplo, o modelo teórico de Lawrence e Tolbert (2007), sobre decisões e comportamentos de carreira, propõe que percepções favoráveis sobre oportunidades de carreira resultam em aumento de comprometimento organizacional, uma vez que os profissionais tenderiam a investir seu tempo, esforço e competências na organização, favorecendo a formação de vínculos afetivos.

Em acréscimo, pesquisas empíricas pregressas reportaram correlações positivas e moderadas entre percepção de oportunidades de carreira, comprometimento afetivo (Crawshaw, van Dick, \& Brodbeck, 2012; Kraimer, Seibert, Wayne, Liden, \& Bravo, 2011) e comprometimento em geral (Chay \& Aryee, 1999). Prince (2003) relata que expectativas de enriquecimento do cargo (isto é, obtenção de atividades mais complexas e autônomas) predizem comprometimento organizacional geral (efeito positivo e moderado). Esses estudos evidenciaram o elo entre comprometimento organizacional e variáveis correlatas ao construto aqui adotado (expectativas de carreira na organização). Combinando tais dados com a fundamentação teórica anterior, é possível derivar a hipótese 3:

Hipótese 3 - Expectativas de carreira na organização relacionam-se positivamente ao comprometimento organizacional afetivo.

No caso da relação de expectativas de carreira e intenção de desligamento, além dos mecanismos inerentes à troca social e à norma da reciprocidade, salienta-se que modelos teóricos sobre rotatividade/desligamento (por exemplo, Mobley, Griffeth, Hand, \& Meglino, 1979) postulam o papel de projeções sobre a carreira/trabalho na formação da intenção de sair da organização. Com efeito, expectativas de carreira elevadas podem incrementar o senso de mutualismo de interesses e dissuadir os indivíduos de planos de desligamento, até porque só continuando na organização as expectativas poderão se materializar em avanços reais na carreira.

Como aponta a literatura especializada em psicologia do tempo, expectativas de sucesso motivam os indivíduos a se engajar em atividades que permitam a realização do futuro desejado (Oettingen, 2012), o que pressupõe a permanência na organização (e menor intenção de desligamento). Assim, espera-se relação negativa entre as variáveis, elevadas (baixas) expectativas para a carreira vinculam-se à baixa (alta) intenção de desligamento.

Do ponto de vista empírico, estudos prévios acusaram correlações negativas e moderadas entre expectativas/ percepções de futuro na carreira e intenção de desligamento (Chay \& Aryee, 1999; Chen, Ployhart, Cooper-Thomas,
Anderson, \& Bliese, 2011). A presente pesquisa testa esse padrão de resultados, mas utilizando uma forma diferente de conceber/aferir o futuro na carreira. Assim, a hipótese 4 aponta que:

Hipótese 4 - Expectativas de carreira na organização relacionam-se negativamente à intenção de desligamento.

\section{Comprometimento Organizacional Afetivo e Intenção de Desligamento}

Ao contrário das hipóteses anteriores, a relação entre comprometimento organizacional e intenção de desligamento/rotatividade já foi bastante estudada na literatura. A quantidade de estudos decorre, em grande parte, da ideia intuitiva de que quanto maior a identificação e o elo afetivo com a organização (comprometimento), menor a intenção de deixá-la. Do ponto de vista empírico, uma das primeiras meta-análises sobre comprometimento (Mathieu \& Zajac, 1990) já revelava a forte associação negativa da variável com intenção de desligamento. Resultados de meta-análise posterioriores (Meyer, Stanley, Herscovitch, \& Topolnytsky, 2002) apontaram na mesma direção (especialmente no caso do comprometimento afetivo) e esse padrão continua a assomar em investigações recentes (Juhdi, Pa'wan, \& Hansaram, 2013). Com base no exposto, a hipótese 5 estipula que:

Hipótese 5 - Comprometimento organizacional afetivo relaciona-se negativamente à intenção de desligamento.

Ao integrar essa hipótese às anteriores, nota-se que o modelo contém mecanismos de mediação. Propõe-se que o efeito das expectativas de futuro organizacional em intenção de desligamento seja totalmente mediado por comprometimento organizacional afetivo e expectativas de carreira, pois não foi localizada, na literatura, justificativa para o efeito direto. Esperam-se, ainda, duas mediações parciais: o efeito das expectativas de futuro organizacional em comprometimento organizacional afetivo seria mediado por expectativas de carreira e o efeito de expectativas de carreira em intenção de desligamento seria mediado por comprometimento organizacional afetivo. Em ambos os casos, não foram encontrados estudos anteriores que indicassem a capacidade das variáveis mediadoras em suprimir integralmente os efeitos diretos das variáveis independentes nas variáveis dependentes.

\section{Variáveis Moderadoras}

Como a literatura sobre futuro na carreira ainda carece de pesquisas sobre moderação (Crawshaw et al., 2012), esta pesquisa testa moderadores para as três relações do modelo, nas quais as expectativas (de futuro organizacional e de carreira) atuam como variáveis antecedentes de comprometimento organizacional e intenção de desligamento (hipóteses H2, H3 e H4). 
Tipo de organização (pública ou privada) foi o primeiro moderador testado. Estudos brasileiros (Salles \& Nogueira, 2006; Sant'anna, Paschoal, \& Gosendo, 2012) sustentam que há diferenças entre os dois ambientes de trabalho no país. Em geral, o funcionalismo público (administração direta, fundações e autarquias) caracteriza-se por maior estabilidade de trabalho, vínculos de longo prazo/relacionais, previsibilidade e por trajetórias de carreira mais limitadas, rígidas e influenciadas por tempo de serviço. $\mathrm{Na}$ iniciativa privada, há menor previsibilidade/estabilidade no ambiente de trabalho, os vínculos tendem a ser de curto prazo/ transacionais, porém existe maior agilidade na trajetória de carreira.

No funcionalismo público, como existe maior previsibilidade quanto ao futuro da organização e da carreira, é provável que a influência das expectativas nas demais variáveis do modelo seja menor do que no setor privado. Por exemplo, a valorização da estabilidade, pelos funcionários públicos, deve minimizar o efeito das expectativas na intenção de desligamento, em oposição à dinâmica do setor privado. De outra parte, o comprometimento organizacional vincula-se mais claramente a variáveis ligadas à carreira (como comprometimento com a carreira e adequação das políticas de promoção) nas empresas privadas, do que no funcionalismo público (Bastos, 1994). No setor privado, como o vínculo indivíduo-organização geralmente é mais fugaz e pragmático, o comprometimento e a permanência na organização dependeriam mais das crenças dos profissionais sobre o futuro da organização e sobre o futuro na carreira. Nessa linha, a hipótese 6 sustenta que:

Hipótese 6 - Tipo de organização modera a relação de expectativas de futuro organizacional e de carreira na organização com comprometimento organizacional afetivo e intenção de desligamento, de forma que a relação entre as variáveis será mais forte no setor privado do que no funcionalismo público.

A segunda variável de moderação advém do referencial teórico de orientação (perspectiva) temporal de Zimbardo e Boyd (1999). Orientação temporal refere-se ao processo não consciente pelo qual os indivíduos organizam e interpretam o fluxo de experiências pessoais/sociais. Há cinco dimensões de orientação temporal (passado-negativo, passado-positivo, presente-hedonista, presente-fatalista e futuro) e a ênfase em determinada dimensão pode atuar como tendência cognitiva capaz de condicionar sistematicamente julgamentos, intenções, decisões e comportamentos dos indivíduos.

Neste artigo, o foco recai na orientação para o futuro. Zimbardo e Boyd (1999) ponderam que indivíduos com altos escores nessa dimensão tendem a planejar e organizar cuidadosamente suas vidas e a enfatizar recompensas/ objetivos futuros (inclusive de carreira), mesmo que esses demandem sacrifícios no presente (adiamento de gratificação). Com base no referencial teórico de Zimbardo e Boyd (1999), é possível supor que, em indivíduos altamente orientados para o futuro, o efeito das expectativas em comprometimento organizacional afetivo e intenção de desligamento seja maior (em módulo) do que nos indivíduos que atribuem menor relevância a tal quadro temporal. Isto é, dada a grande importância do futuro, as expectativas (negativas ou positivas) influenciariam mais fortemente as variáveis orientadas para o presente. Assim, a hipótese 7 propõe que:

Hipótese 7 - Orientação para o futuro modera a relação de expectativas de futuro organizacional e de carreira na organização com comprometimento organizacional afetivo e intenção de desligamento, de forma que a relação entre as variáveis será mais forte em indivíduos com elevada orientação para o futuro do que nos demais.

\section{MÉTODO}

\section{Participantes}

Na amostra $(n=370)$, a idade média foi de 42,04 anos $(\mathrm{DP}=12,42), 50 \%$, eram homens e todos apresentavam escolaridade igual/superior ao nível médio. Os profissionais, oriundos de diversos estados e regiões do Brasil, trabalhavam em várias organizações, com preponderância do setor privado $(49 \%)$ e do funcionalismo público (36\%). O recrutamento dos participantes foi realizado por consultoria especializada. Esta foi contratada especificamente para realizar essa tarefa e sua atuação se restringiu ao referido recrutamento. Um incentivo de cerca de R $\$ 15$ reais foi concedido pela participação na pesquisa. Para $\alpha=0,05$, essa amostra fornece poder estatístico superior a $80 \%$ nos modelos relatados na seção de resultados, considerando $\varepsilon_{0} \leq 0,05$ e $\varepsilon_{\mathrm{a}}=0,08$, respectivamente, como hipótese nula e valor testado do Root Mean Square Error of Approximation (RMSEA).

\section{Instrumentos}

Expectativas de carreira na organização e de futuro organizacional. Foram empregadas a Escala de Expectativas de Futuro Organizacional (EEFO; Vasconcellos \& Neiva, 2016) e a versão reduzida da Escala de Expectativas de Carreira na Organização (EECO; Vasconcellos \& Neiva, 2017). As assertivas da EEFO remetem à situação da organização no futuro (exemplo: "O ambiente de trabalho será aperfeiçoado pela gestão da organização") e as da EECO aludem à situação da carreira do indivíduo naquela organização no futuro (exemplo: "Serei um profissional bem-sucedido na minha carreira"), em ambos os casos, de forma positivada.

Nas escalas, os participantes assinalam, em escala de sete pontos, a probabilidade dessa situação ocorrer cinco anos à frente (1=nada provável até $7=$ extremamente provável). 
A EEFO apresenta nove itens distribuídos em dois fatores: Gestão e Ambiente Organizacional (quatro itens; $\alpha=0,91$ ) e Efetividade Organizacional (cinco itens; $\alpha=0,91$ ). A EECO conta com 10 itens, divididos em dois fatores: Conquistas Profissionais (seis itens; $\alpha=0,91$ ) e Relação Carreira-Vida Pessoal (quatro itens; $\alpha=0,91$ ).

Comprometimento organizacional afetivo. Foram utilizados cinco itens $(\alpha=0,87)$ da escala de comprometimento afetivo, de Menezes e Bastos (2011), selecionados a partir de suas cargas fatoriais e ligação semântica com o construto. "Sinto que existe uma forte ligação afetiva entre mim e esta organização" é um exemplo de item. Os itens foram respondidos em escala de concordância (cinco pontos).

Intenção de desligamento. A variável foi medida pela Escala de Intenção de Rotatividade (Siqueira, Gomide, Oliveira, \& Filho 2014). Cho et al. (2009) atestam que intenção de desligamento e intenção de rotatividade (turnover intention) são usualmente conceituados/utilizados de forma intercambiável na literatura. De fato, os itens dessa escala espelham o conceito de intenção de desligamento aqui adotada. Seus três itens $(\alpha=0,93)$ foram respondidos por intermédio de escala de frequência $(1=$ nunc $a$ até $5=$ sempre $)$. Exemplo de item: "Penso em sair da organização onde trabalho".

Orientação para o futuro. A variável foi mensurada pelo fator Orientação ao Futuro, da Escala de Orientação Temporal (Souza, 2013). Foram selecionados os seis itens com as maiores cargas fatoriais do fator $(\alpha=0,78)$. "Tenho projetos e planos de futuro bem definidos" é um exemplo de item. A escala foi respondida por meio de escala de concordância (cinco pontos).

Além dessas variáveis, os respondentes indicavam seu sexo, idade, tempo de organização e tipo de organização empregadora. Como as variáveis foram aferidas no mesmo questionário, o viés de método comum foi uma preocupação. Para minimizar esse risco, foram seguidas as recomendações de Podsakoff, MacKenzie, Lee e Podsakoff (2003), de garantir o anonimato das respostas e de separar as variáveis no questionário tanto psicológica (ao adotar introduções customizadas para cada variável), quanto metodologicamente (usando escalas com âncoras e número de pontos diferentes).

\section{Procedimentos}

Os potenciais respondentes receberam um link para pesquisa (taxa de resposta de $25 \%$ ), no qual constavam as instruções e o questionário. Observando os preceitos éticos de pesquisa, as instruções contavam com informações sobre o objeto/propósito de estudo, identificação do pesquisador-líder e sua instituição, e-mail de contato e garantia de confidencialidade. A participação na pesquisa foi voluntária e anônima, ocorrendo com consentimento prévio dos respondentes. Estes tinham direito de desistir de sua participação a qualquer momento.

\section{Análise de Dados}

A análise dos dados compreendeu a avaliação do modelo de mensuração (via análise fatorial confirmatória) e do modelo estrutural (para testar as hipóteses). Em ambos os casos, os construtos expectativas de futuro organizacional e expectativas de carreira na organização utilizaram as médias de seus fatores como variáveis empíricas. Essa estratégia de agregação é comum na literatura, pois favorece o surgimento de variáveis com distribuição mais intervalar, normal e confiável. Os modelos foram calculados no programa AMOS 18, pelo método de estimação máxima verossimilhança. Como os dados não exibiram normalidade multivariada (aferida pelo teste de Mardia), recorreu-se a um bootstrapping com 500 subamostras para avaliar a estabilidade dos parâmetros estimados.

No teste de hipóteses, foram observados o sinal, magnitude e significância dos coeficientes, além do ajuste geral dos modelos. Além do RMSEA, os seguintes índices foram considerados: GFI (Goodness-of-Fit Index), CFI (Comparative Fit Index), SRMR (Standardized Root Mean Residual) e a diferença entre os valores de $\chi^{2}$ (qui-quadrado) para avaliar modelos alternativos. Comparou-se o modelo proposto a modelos alternativos, haja vista que tal prática constitui teste mais rigoroso do que a análise isolada de seu ajuste (Hair, Black, Babin, Anderson, \& Tatham, 2010). Análises sobre modelos alternativos, mediação e moderadores seguiram as orientações de Hair et al. (2010).

As hipóteses sobre moderação foram examinadas por análise multigrupo. Os dois grupos comparados na variável tipo de organização foram organizações do funcionalismo público e organizações privadas. Em orientação para o futuro, também foram comparados dois grupos, um com indivíduos com escores no fator acima da mediana e outro com indivíduos com escores abaixo da mediana. Na prática, todos no primeiro grupo obtiveram escore igual ou maior que 4,00 , intervalo que abrange os pontos de concordância da escala (o grupo foi denominado como alta orientação para o futuro). E todos no segundo grupo obtiveram valor inferior a 4,00, intervalo que engloba os pontos de neutralidade e discordância da escala (o grupo foi denominado como média/ baixa orientação para o futuro).

Outros critérios de divisão de grupos presentes na literatura foram preteridos, visto que formariam pelo menos um grupo com tamanho amostral pequeno, reduzindo o poder estatístico e dificultando o uso de modelos estruturais. Testes $t$ apontaram que os dois grupos gerados diferem fortemente $(p<0,001, d>0,80)$ nos escores do fator, o que fornece maior segurança para o teste de moderação. 


\section{RESULTADOS}

\section{Estatísticas Descritivas e Análise Fatorial Confirmatória}

A Tabela 1 exibe as estatísticas descritivas e correlações das variáveis investigadas. As expectativas de carreira e de futuro organizacional correlacionaram-se positivamente com comprometimento organizacional afetivo (efeitos moderados e fortes) e negativamente com intenção de desligamento (efeitos moderados). Esses dados vieram em linha com o esperado.

A adequação da mensuração dos construtos foi avaliada por análise fatorial confirmatória e testes de modelos alternativos (Tabela 2). O modelo proposto (quatro fatores) exibiu bom ajuste em todos os índices. Todas as cargas fatoriais foram significativas $(p<0,01)$ e superiores a 0,55 . Não foi necessário realizar nenhuma ligação entre os termos de erro das variáveis empíricas. Os modelos alternativos (um, dois e três fatores) foram estatisticamente inferiores (pela diferença de $\chi^{2}$ ) e apresentaram índices de ajuste piores do que o modelo proposto. Assumiu-se, então, modelo de mensuração proposto como adequado.

As médias das cargas fatoriais das subamostras do bootstrapping foram praticamente idênticas às cargas fatoriais do modelo proposto (a diferença máxima não atingiu 0,01 ), com baixo erro padrão (o máximo foi 0,05 ). Esses resultados demonstram a estabilidade das cargas e fornecem evidência sobre a adequação do processo de estimação do modelo.

Tabela 1

Médias, Desvios-padrão e Correlações das Variáveis do Modelo de Investigação Proposto

\begin{tabular}{|c|c|c|c|c|c|c|c|}
\hline Variáveis & Média (DP) & 1 & 2 & 3 & 4 & 5 & 6 \\
\hline $\begin{array}{l}\text { 1. ECO Carreira } \\
\text { e Vida pessoal }\end{array}$ & $4,85(1,41)$ & - & & & & & \\
\hline $\begin{array}{l}\text { 2. ECO } \\
\text { Conquistas } \\
\text { Profissionais }\end{array}$ & $4,61(1,38)$ & 0,63 & - & & & & \\
\hline $\begin{array}{l}\text { 3. EFO Gestão } \\
\text { e Ambiente } \\
\text { Organizacional }\end{array}$ & $4,60(1,31)$ & 0,47 & 0,67 & - & & & \\
\hline $\begin{array}{l}\text { 4. EFO } \\
\text { Efetividade } \\
\text { Organizacional }\end{array}$ & $4,99(1,29)$ & 0,43 & 0,67 & 0,76 & - & & \\
\hline $\begin{array}{l}\text { 5. Intenção de } \\
\text { Desligamento }\end{array}$ & $2,67(1,15)$ & $-0,26$ & $-0,43$ & $-0,35$ & $-0,36$ & - & \\
\hline 6. COA & $3,70(0,84)$ & 0,35 & 0,53 & 0,49 & 0,52 & $-0,47$ & - \\
\hline $\begin{array}{l}\text { 7. Orientação } \\
\text { para Futuro }\end{array}$ & $3,79(0,70)$ & 0,21 & 0,22 & 0,14 & 0,15 & $-0,09$ & 0,24 \\
\hline
\end{tabular}

Nota. $\mathrm{ECO}=$ Expectativas de Carreira na Organização; EFO=Expectativas de Futuro Organizacional; COA=Comprometimento Organizacional Afetivo. Todas as correlações são significativas $(p<0,01)$, exceto no caso da correlação entre orientação para futuro e intenção de desligamento $(r=-0,09, p=0,09)$.

Tabela 2

Índices de Ajuste dos Modelos na Análise Fatorial Confirmatória

\begin{tabular}{lccccccccc}
\hline Modelo & $\boldsymbol{\chi}^{2}$ & $\boldsymbol{g}$ & GFI & CFI & RMSEA & IC $_{\text {RMSEA }}$ & SRMR & $\Delta \boldsymbol{\chi} \mathbf{2}$ \\
\hline $\begin{array}{l}\text { Quatro fatores } \\
\text { (proposto) }\end{array}$ & $137,54^{* *}$ & 48 & 0,94 & 0,97 & 0,07 & {$[0,06-0,09]$} & 0,04 & - \\
Três fatores $^{\mathrm{a}}$ & $206,29 * *$ & 51 & 0,91 & 0,95 & 0,09 & {$[0,08-0,10]$} & 0,04 & $68,75^{* *}$ \\
Dois fatores $^{\mathrm{b}}$ & $863,13^{* *}$ & 53 & 0,70 & 0,72 & 0,20 & {$[0,19-0,22]$} & 0,10 & $725,59 * *$ \\
Um fator & $1203,81^{* *}$ & 54 & 0,62 & 0,61 & 0,24 & {$[0,23-0,25]$} & 0,13 & $1066,27 * *$ \\
\hline
\end{tabular}

Nota. GFI=Goodness-of-Fit Index; CFI=Comparative Fit Index; RMSEA=Root Mean Square Error of Approximation; $\mathrm{IC}_{\mathrm{RMSEA}}$ Intervalo de Confiança 90\% do RMSEA; SRMR=Standardized Root Mean Residual.

${ }^{a}$ Expectativas de carreira e de futuro organizacional como um único fator.

${ }^{b}$ Expectativas de carreira e de futuro organizacional como um único fator e intenção de desligamento e comprometimento organizacional afetivo como um único fator.

$* * p<0,01$. 
Verificou-se, então, o risco de viés de método comum. As técnicas para detectar tal viés ainda são limitadas/imprecisas, de sorte que não há consenso na literatura sobre qual delas é a melhor (Podsakoff et al., 2003; Richardson, Simmering, \& Sturman, 2009). Nesse cenário, optou-se, aqui, pelo difundido teste de Harman. Nesse teste, executa-se uma análise fatorial exploratória, sem rotação com todos os itens do modelo. Se o primeiro fator explicar mais de $50 \%$ da variância, há indícios de viés (Podsakoff et al., 2003), fato que não se consumou no modelo proposto. Recentemente, uma variante dessa técnica ganhou espaço na literatura: gerase um modelo de fator único na análise fatorial confirmatória e seu ajuste é avaliado. Como evidencia a Tabela 2, tal modelo manifestou péssimo ajuste. Logo, não parece existir risco elevado de viés de método comum.

\section{Modelo Estrutural e Teste de Hipóteses}

O modelo estrutural proposto registrou bons índices de ajuste: $\chi^{2}(g l)=137,56(49), p<0,01, \mathrm{GFI}=0,94, \mathrm{CFI}=0,97$, RMSEA [IC 90\%] $=0,07[0,06-0,08]$ e SRMR $=0,04$. As estimativas dos coeficientes padronizados estão na Figura 2 e todas foram estatisticamente significativas $(p<0,01)$. As hipóteses 1 e 2 estabeleciam que as expectativas de futuro organizacional relacionavam-se positivamente às expectativas de carreira na organização e ao comprometimento organizacional afetivo. Os resultados suportaram ambas as hipóteses.

As hipóteses 3 e 4 previam que expectativas de carreira na organização relacionavam-se positivamente ao comprometimento organizacional afetivo e negativamente à intenção de desligamento. Os coeficientes estimados foram consistentes com ambas as hipóteses. A hipótese 5 , que apontava a relação negativa de comprometimento organizacional afetivo com intenção de desligamento, também foi suportada pelos resultados. O modelo explicou
$41 \%$ da variância de comprometimento organizacional afetivo e $32 \%$ de intenção de desligamento.

Ainda que todas as hipóteses tenham sido corroboradas, o modelo proposto foi cotejado a um modelo alternativo. $\mathrm{O}$ modelo alternativo testado adicionou ao modelo proposto a ligação direta entre expectativas de futuro organizacional e intenção de desligamento. O coeficiente padronizado foi extremamente baixo $(0,01)$ e não significativo $(p>0,05)$. A diferença no qui-quadrado entre os modelos, $\Delta \chi^{2}(1)=0,02$, também não foi significativa $(p>0,05)$, apontando que a inclusão da ligação não melhorou o modelo proposto. Os dados sugerem, portanto, que a relação entre os dois construtos na análise fatorial confirmatória $(r=-0,42 ; p<0,01)$ foi totalmente mediada por expectativas de carreira na organização e comprometimento organizacional afetivo. Assim, o modelo proposto originalmente foi assumido como o mais adequado e utilizado na análise de moderadores.

O teste de moderadores foi realizado por análise multigrupo, conforme indicado por Hair et al. (2010). Tais autores apontam, como pré-condição para esse teste, a necessidade de duas formas de invariância entre grupos no modelo de mensuração. A primeira (invariância de configuração) é assumida se os grupos conservam a mesma estrutura fatorial em um modelo sem restrições, no qual as cargas fatoriais são livremente estimadas para cada grupo (Hair et al., 2010; Kline, 2011). O ajuste desse modelo é avaliado, bem como a equivalência dos grupos quanto ao número de fatores e alocação dos itens em cada fator. A segunda (invariância métrica) é medida por meio de modelo aninhado (nested model) ao modelo sem restrições, no qual a igualdade das cargas fatoriais entre os dois grupos é imposta (Hair et al., 2010; Kline, 2011). Assume-se invariância métrica quando não há diferença estatisticamente significativa entre os valores de $\chi^{2}$ do modelo sem restrições e do modelo que impõe a igualdade das cargas entre os grupos, sinal que tais restrições não pioram o modelo.

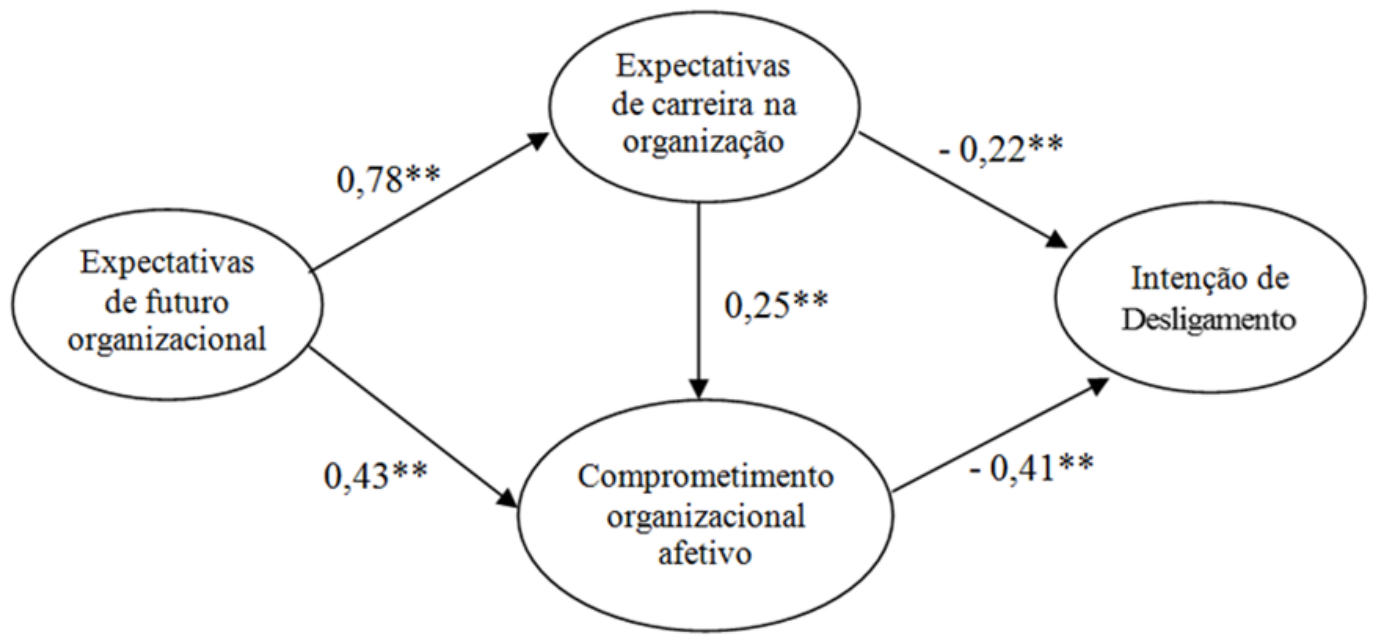

Figura 2. Coeficientes padronizados do modelo estrutural proposto

$* * p<0,01$. 
Os dois pares de grupos (organizações do funcionalismo público versus privadas e alta versus média/baixa orientação para o futuro) foram verificados quanto à sua invariância no modelo de mensuração. Nos dois pares, as estruturas fatoriais foram iguais e os índices de ajuste dos modelos sem restrições foram aceitáveis (GFI $\geq 0,89, \mathrm{CFI} \geq 0,95$ e RMSEA $\leq 0,06$ ), ou seja, há indicação de invariância de configuração. Os dados também suportaram invariância métrica nos dois pares: a diferença de ajuste $\left(\Delta \chi^{2}\right)$ entre os modelos com e sem restrição de igualdade nas cargas fatoriais registrou $p>0,05$.

O próximo passo foi testar efetivamente os moderadores. Seguindo as hipóteses estabelecidas, três relações entre construtos foram avaliadas: a) expectativas de futuro organizacional e comprometimento organizacional afetivo; b) expectativas de carreira na organização e comprometimento organizacional afetivo e c) expectativas de carreira na organização e intenção de desligamento. Embora o foco esteja nessas três relações, os demais coeficientes estruturais também foram analisados de forma complementar. Partindo dos modelos que asseguravam a invariância métrica, avaliou-se a moderação pela diferença de qui-quadrado entre o modelo estrutural sem restrições (no qual os coeficientes estruturais dos dois grupos de cada variável moderadora foram livremente estimados) e os modelos estruturais com a restrição de igualdade entre grupos em cada coeficiente, um de cada vez.

O primeiro moderador analisado foi tipo de organização. Entre os cinco modelos estruturais restritos gerados (um para cada coeficiente estrutural), apenas o que igualou organizações do funcionalismo público e privadas na ligação de expectativas de carreira, com intenção de desligamento, exibiu diferença estatística significativa com o modelo sem restrições: $\Delta \chi^{2}(1)=5,17 ; p=0,02$. É um indício de moderação. Ademais, quando livremente estimado entre os grupos, o efeito (coeficiente padronizado) de expectativas de carreira em intenção de desligamento foi moderado/ significativo nas organizações privadas $(-0,38 ; p<0,01) \mathrm{e}$ fraco/não significativo nas públicas $(-0,13 ; p=0,22)$. Os demais coeficientes das organizações privadas e públicas são estatisticamente similares e muito próximos em magnitude. Considera-se a hipótese 6 parcialmente suportada.

No outro moderador examinado, orientação para o futuro, apenas o modelo que impôs igualdade entre grupos no coeficiente que liga expectativas de carreira ao comprometimento organizacional afetivo apresentou diferença estatística significativa com o modelo sem restrições: $\Delta \chi^{2}(1)=4,64 ; p=0,03$. Quando livremente estimado entre os grupos, o efeito (coeficiente padronizado) de expectativas de carreira em comprometimento organizacional foi moderado e significativo no grupo com média/baixa orientação para o futuro $(0,41 ; p<0,01) \mathrm{e}$ ausente/não significativo no grupo com alta orientação para o futuro $(0,00 ; p=0,99)$.

Os dados sugerem o papel moderador de orientação para o futuro na relação entre essas variáveis. Porém, o grupo com maior coeficiente foi o de média/baixa orientação para o futuro (isto é, na direção oposta da prevista), portanto, a hipótese 7 não foi sustentada. A comparação dos demais modelos com coeficientes estruturais restritos com o modelo sem restrição não acusou nenhuma diferença de qui-quadrado significativa (considerando $\alpha=0,05$ ).

\section{DISCUSSÃO}

Os resultados suportaram as hipóteses, exceto no caso das hipóteses sobre moderadores. O modelo estrutural obtido sugere que as expectativas dos indivíduos sobre suas carreiras na organização dependem fortemente das expectativas nutridas sobre o futuro da própria organização. Esse dado reforça, por meio de um modelo quantitativo, pesquisas qualitativas anteriores, que identificaram tal ligação no discurso dos profissionais (Duarte \& Silva, 2013; Margolis \& Hansen, 2003; Ullrich et al., 2005) e sugerem que estes atribuem grande peso às contingências do ambiente organizacional na formação das expectativas de carreira. $\mathrm{O}$ modelo indica, ainda, que expectativas mais favoráveis de futuro organizacional e de carreira se vinculam a níveis maiores de comprometimento afetivo. É possível que o vínculo com a organização derive de experiências passadas ou atuais dos indivíduos no trabalho. Este estudo sugere que expectativas sobre futuro também atuam na produção desse vínculo.

As análises apontaram, igualmente, que quanto mais favoráveis forem as expectativas de carreira, menor será a intenção de desligamento, contribuindo, nesse sentido, para além de comprometimento organizacional, o mais consistente preditor dessa variável (Meyer et al., 2002). A relação negativa e moderada entre projeções de futuro na carreira e intenção de rotatividade/desligamento foi identificada previamente (Chay \& Aryee, 1999; Chen et al., 2011). Os resultados endossaram esse padrão, contudo, expandiram a literatura ao conceituar o futuro na carreira por meio de expectativas, medi-lo com um instrumento mais abrangente e testá-lo em um modelo que contava com comprometimento organizacional.

O efeito das expectativas de carreira em intenção de desligamento foi mais robusto nas organizações privadas do que nas públicas. Nessas últimas, a intenção de sair/ permanecer na organização parece menos dependente do futuro na carreira, possivelmente em função da influência do apreço pela estabilidade e da construção de vínculos relacionais de longo prazo. No setor privado, a relação com a organização tende a ser mais transacional e pragmática: logo, a intenção de desligamento sofreria efeito mais direto das 
expectativas de carreira, que refletem crenças sobre futuras contrapartidas, obtidas junto à organização.

Contrariando a hipótese 7 , o efeito das expectativas de carreira no comprometimento afetivo foi maior no grupo com média/baixa orientação para o futuro do que no grupo com alta orientação para o futuro (nesse último grupo, o efeito foi nulo). É possível que indivíduos com alta orientação para o futuro planejem/organizem o futuro cuidadosamente e provavelmente atribuam conquistas futuras na carreira aos seus próprios méritos/esforços (e não à organização). Sentem-se, então, menos imersos no mecanismo de troca social com a organização e menos obrigados a retribuir com comprometimento. Indivíduos com média/baixa orientação para o futuro planejam e se esforçam menos para obter um porvir favorável na carreira. Esse passaria, então, a ser interpretado mais como fruto das práticas organizacionais do que como resultado de esforço/planejamento pessoal, exigindo, assim, mais reciprocidade (na forma de comprometimento) na relação com a organização.

Esta pesquisa contribui para a literatura ao valorizar a dimensão temporal na compreensão da dinâmica indivíduoorganização, abordagem residual na academia (Lee \& Liebenau, 1999; Sonnentag, 2012). Em especial, explicita o entrelaçamento do presente com o futuro na predição de comprometimento organizacional afetivo e intenção de desligamento. Nesse sentido, expectativas favoráveis para a carreira e para o ambiente organizacional (futuro positivo antecipado) parecem retroagir temporalmente sobre aquelas variáveis, estimulando nos profissionais vínculos mais profundos com a organização.

Em termos práticos, os resultados sugerem que a gestão de pessoas deve atentar para as crenças dos profissionais sobre seu próprio futuro e sobre o futuro organizacional. Em mercados de trabalho com disputa por talentos, a presença de expectativas favoráveis pode garantir a retenção de bons quadros e a elevação do comprometimento. Ainda que a intenção de desligamento nem sempre se traduza em efetivo desligamento, certamente aumenta o risco de evasão de profissionais. Torna-se relevante aferir, por meio de pesquisas institucionais, como estão as expectativas e investir em práticas capazes de fomentar expectativas positivas, como a criação de trilhas de carreiras (futuro profissional) e a comunicação adequada dos planos de negócios e visão da organização (futuro organizacional).

Entre as limitações da pesquisa estão: o uso exclusivo de medidas de autorrelato (muito suscetíveis à desejabilidade social), a não aleatoriedade da amostra (que pode gerar desequilíbrio na composição dos grupos de respondentes), o caráter transversal da coleta de dados e a impossibilidade de testar no modelo outras variáveis importantes, tais como percepção de alternativas de emprego e o efetivo desligamento dos profissionais. Com efeito, os resultados relatados não podem ser assumidos estritamente como relações causais entre variáveis. Pesquisas futuras podem incluir tais variáveis no modelo e assumir desenho longitudinal, oportunizando testes mais abrangentes e complexos.

A relação com o futuro marca a subjetividade humana de diversas formas. No mundo organizacional, pouco se sabe sobre o modo como as expectativas influenciam o momento atual dos profissionais. Mesmo com suas limitações, esperase que este artigo abra veredas para novas pesquisas nesse instigante (e pouco explorado) campo de estudos.

\section{REFERÊNCIAS}

Bandura, A. (1977). Self-efficacy: Toward a unifying theory of behavioral change. Psychological Review, 84, 191-215.

Bastos, A. V. (1994). Comprometimento organizacional: Seus antecedentes em distintos setores da administração e grupos ocupacionais. Temas em Psicologia, 1, 73-90.

Blau, P. (1964). Exchange and power in social life. New York: John Wiley.

Carver, C. S., \& Scheier, M. F. (2017). Optimism, coping, and wellbeing. In C. L. Cooper \& J.C. Quick (Eds), The Handbook of stress and health (pp. 400-414). UK: John Wiley

Chay, Y., \& Aryee, S. (1999). Potential moderating influence of career growth opportunities on careerist orientation and work attitudes: Evidence of the protean career era in Singapore. Journal of Organizational Behavior, 20(5), 613-623.

Chen, G., Ployhart, R., Cooper-Thomas, H., Anderson, N., \& Bliese, P. (2011). The power of momentum: A new model of dynamic relationships between job satisfaction change and turnover intentions. Academy of Management Journal, 54, 159-181.

Chiu, W. (2002). Do types of economic ownership matter in getting employees to commit? International Journal of Human Resource Management, 13, 865-882.

Cho, S., Johanson, M., \& Guchait, P. (2009). Employees intent to leave: A comparison of determinants of intent to leave versus intent to stay. International Journal of Hospitality Management, 28, 374-381.

Crawshaw, J., van Dick, R., \& Brodbeck, F. C. (2012). Opportunity, fair process and relationship value: Career development as a driver of proactive work behaviour. Human Resource Management Journal, 22(1), 4-20.

Duarte, M., \& Silva, A. (2013). A reconstrução da carreira em ambientes de trabalho em transformação. Organizações \& Sociedade, 20, 699-715.

Gouldner, A. (1960). The norm of reciprocity: A preliminary statement. American Sociological Review, 25, 161-178.

Greenhaus, J. \& Kossek, E. (2014). The contemporary career: A work-home perspective. Annual Review of Organizational Psychology and Organizational Behavior, 1, 361-388.

Hair, J., Black, W., Babin, B., Anderson, R. \& Tatham, R. (2010). Multivariate data analysis $\left(7^{\mathrm{a}}\right.$ ed.). Upper Saddle River, NJ: Prentice Hall.

Kline, R. (2011). Principles and practice of structural equation modeling. New York: Guilford.

Kraimer, M., Seibert, S., Wayne, S., Liden, R., \& Bravo, J. (2011). Antecedents and outcomes of organizational support for development: The critical role of career opportunities. Journal of Applied Psychology, 96, 485-500. 
Juhdi, N., Pa'wan, F., \& Hansaram, R. (2013). HR practices and turnover intention: Mediating roles of organizational commitment and organizational engagement in a selected region in Malaysia. The International Journal of Human Resource Management, 24, 3002-3019.

Lawrence, B., \& Tolbert, P. (2007). Organizational demography and individual careers: structure, norms, and outcomes. In $\mathrm{H}$. Gunz, \& M. A. Peiperl (Eds.), Handbook of career studies (pp. 399-421). Thousand Oaks, CA: Sage.

Lee, H., \& Liebenau, J. (1999). Time in organizational studies: Towards a new research direction. Organization Studies, 20, 1035-1058.

Magalhães, M., \& Bendassolli, P. (2013). Desenvolvimento de carreiras nas organizações. In L. Borges \& M. Mourão (Orgs.). O trabalho e as organizações (pp. 433-464). Porto Alegre: Artmed.

Margolis, S., \& Hansen, C. D. (2003). Visions to guide performance: A typology of multiple future organizational images. Performance Improvement Quarterly, 16(4), 40-58.

Mathieu, J., \& Zajac, D. (1990). A review and meta-analysis of the antecedents, correlates, and consequences of organizational commitment. Psychological Bulletin, 108, 171-194.

Menezes, I. \& Bastos, A. (2011). Comprometimento organizacional atitudinal: Estudo empírico sobre a dimensionalidade do construto. Estudos de Psicologia (Campinas), 28, 463-474.

Meyer, J., Stanley, D., Herscovitch, L., \& Topolnytsky, L. (2002). Affective, continuance, and normative commitment to the organization: A meta-analysis of antecedents, correlates and consequences. Journal of Vocational Behavior, 61, 20-52.

Mitchell, T., Burch, T., \& Lee, T. (2014). The need to consider time, level, and trends: A turnover perspective. Journal of Organizational Behavior, 35(2), 296-300.

Mobley, W., Griffeth, R., Hand, H., \& Meglino, B. M. (1979). Review and conceptual analysis of the employee turnover process. Psychological Bulletin, 86, 493-522.

Mossholder, K., Settoon, R, Harris, S., \& Armenakis, A. (1995). Measuring emotion in open-ended survey responses: An application of textual data analysis. Journal of Management, $21(2), 335-355$.

Oettingen, G. (2012). Future thought and behaviour change. European Review of Social Psychology, 23, 1-63.

Oettingen, G., \& Mayer, D. (2002). The motivating function of thinking about the future: expectations versus fantasies. Journal of Personality and Social Psychology, 83, 1198-1212.

Podsakoff, P., MacKenzie, S., Lee, J., \& Podsakoff, N. (2003). Common method biases in behavioral research: a critical review of the literature and recommended remedies. Journal of Applied Psychology, 88(5), 879-903.

Prince, J. B. (2003). Career opportunity and organizational attachment in a blue-collar unionized environment. Journal of Vocational Behavior, 63(1), 136-150.

Richardson, H. A., Simmering, M. J., \& Sturman, M. C. (2009). A tale of three perspectives: Examining post hoc statistical techniques for detection and correction of common method variance. Organizational Research Methods, 12(4), 762-800.

Salles, D., \& Nogueira, M. (2006). Carreiras no serviço público federal: Antigos dogmas, novas perspectivas. In M. Balassiano \& I. Costa (Orgs.). Gestão de carreiras: Dilemas e perspectivas (pp.134-149). Rio de Janeiro: Editora Atlas.

Sant'anna, L., Paschoal, T., \& Gosendo, E. (2012). Bem-estar no trabalho: Relações com estilos de liderança e suporte para ascensão, promoção e salários. Revista de Administração Contemporânea, 16, 744-764.

Seligman, M. E., Railton, P., Baumeister, R. F., \& Sripada, C. (2013). Navigating into the future or driven by the past. Perspectives on Psychological Science, 8(2), 119-141.

Siqueira M., Gomide Jr., S., Oliveira, A., \& Filho, A. (2014). Intenção de rotatividade. In M. Siqueira (Org.), Novas medidas do comportamento organizacional (pp. 209-216). Porto Alegre: Artmed.

Sonnentag, S. (2012). Time in organizational research: Catching up on a long neglected topic in order to improve theory. Organizational Psychology Review, 2(4), 361-368.

Souza, M. (2013). O uso do crédito pelo consumidor: percepções multifacetadas de um fenômeno intertemporal (Dissertação de Mestrado). Disponível em http://repositorio.unb.br/ handle/10482/13255? mode=full.

Ullrich, J., Wieseke, J., \& van Dick, R. (2005). Continuity and change in mergers and acquisitions. Journal of Management Studies, 42(8), 1549-1569.

Vasconcellos, V. C. \& Neiva, E. R. (2016). Escala de expectativas de carreira na organização: Desenvolvimento e evidências de validade. Psico-USF, 21, 245-257.

Vasconcellos, V. C., \& Neiva, E. R. (2017). Escala de Expectativas de Futuro Organizacional: Desenvolvimento e evidências de validade. Revista Eletrônica de Administração, 23, 58-82.

Vroom, V. H. (1964). Work and motivation. New York: Wiley.

Zimbardo, P., \& Boyd, J. (1999). Putting time in perspective: A valid, reliable individual-differences metric. Journal of Personality and Social Psychology, 77(6), 1271-1288. 